\title{
Another New Design for an Old Map
}

\author{
Kenneth Field ${ }^{\mathrm{a}, *}$, William Cartwright $\mathrm{AM}^{\mathrm{b}}$ \\ aEsriInc,kfield@esri.com \\ ${ }^{b}$ RMIT Melbourne,william.cartwright@rmit.edu.au \\ * Corresponding author
}

Keywords: schematic, map design, cartography, subway map, map

\begin{abstract}
:
Harry Beck's map of the London Underground is iconic, seemingly to the point that attempts to create a new map are almost doomed to failure from the outset. It is the template for many subsequent subway maps and the subway map that is used in London today. Many alternatives have emerged but most rework the basic idea and reconfigure around a style (e.g. London underground styled as a Vignelli New York subway map), structure (e.g. focusing on geometric shapes such as circles or hexagons on which to hang the network) or memes (e.g. use of the map for alternative datasets) $[1,3]$.
\end{abstract}

Yet the network has grown in ways that Beck might never have imagined [4]. There are many more lines and stations in 2019 than there were some 86 years ago. The congested areas are different than they were. The spaces in-between have morphed. Yet the basic principles upon which Beck fashioned his map have remained unchanged [2]. Lines are horizontal, vertical and at $45^{\circ}$ angles. Stations are shown as short ticks, interchanges as circles and connectors. The modern map is cluttered and, arguably, not the best way to help people navigate a complex city.

Here, we've started from scratch. The only nod to the current map is in the colours we use to denote the lines, and the typeface. Both are utterly intertwined with the design that has been a trademark for the map. These are the elements we keep. For the rest, we sketch out a new map, and define our parameters as we go along; as we sketch out a new map.

One of Beck's key tenets was that the above ground detail was relatively unimportant for the traveler. Whilst this still resonates, one might argue that people's navigation is between places above ground [4]. For instance, they might wish to travel between the London Eye and London Zoo. Yet the closest stations do not bear the names of those destinations, and many more suffer similarly. We wanted to make a map that had space for above ground detail too. To provide visual anchors to assist in navigation between points of interest and not just stations with, often, names that might seem rather abstract, particularly to the visitor.

Most re-workings of the map are digital and have an entirely digital workflow. They often start with the current map and simply tweak it. This in itself may be a constraining factor on exploring new designs. Instead, we have gone back to the drawing board, quite literally, and constructed a peg board. This provides a blank canvas and a framework to reconstruct the linear cartogram in an iterative sense, laying down lines and then modifying their position as additions are made. It's an inherently manual process, physically sketching as Beck did in his original diagrams [2, 6, Figure 1].

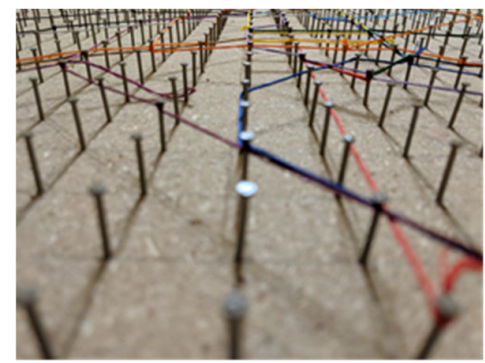

Figure 1: The new map's peg board

The peg board is constructed of 800 nails laid out in a regular 1 inch grid. Coloured thread is used to lay down the lines. The task of building the new map, physically, took around 20 hours. What emerged was a map that bears familiarity to the current map yet which veers markedly from its structure.

Key aspects of the new map are:

- a closer match to geographical relationships between stations;

- reorientation of lines to more closely follow their geographical route;

- several horizontal parallel lines that anchor the map;

- replacement of the historic flask shape for the Circle line, replaced by a diamond to represent part of the Northern line as a key visual shape in the map; 
- increased exaggeration of the central area and relative reduction of the extremities

- the River Thames is drawn geographically.

The peg board was photographed in high resolution and then imported into ArcGIS Pro and georeferenced. A set of styles was developed to provide repeatable digital assets from which to create the map. The reference image of the peg board therefore became a way to trace (digitize) the reconstructed map using the real geography and the River Thames as geographical elements.

A minimum of 1 inch was kept between each adjacent interchange at a minimum. While other stations are located between them, this space was increased where necessary. Many lines are now illustrated in parallel since their true location underground is largely irrelevant to navigation. This allows considerably more space on the map to emerge, in which the above ground points of interest would be located. To accommodate this new approach, a new approach to symbology was designed for individual stations and interchanges. By positioning all station detail within the line itself, the map also becomes cleaner and additional white space for labels and point of interest symbology emerges.

A preliminary version of the new map has been created. It takes a traditional planimetric form that provides a reorganized, cleaner result but which also allows for the inclusion of more detail to help people navigate between points of interest instead of between stations. The inclusion of additional detail has been achieved by using an isometric projection. This equalizes scale across the map so the background (top of the map) does not suffer from being seen smaller than detail in the foreground (bottom of the map). This technique has been used to huge success in maps made by Herman Bollman and Constantine Anderson of New York City [7]. The intent here is to render the network in an isometric form and position 3D symbols of important landmarks and points of interest into the map. The size of the landmarks is scaled to allow minimum occlusion of linework. In isometric view, there's an opportunity to explore alternative label placement too.

In a previous paper [1, p358] we concluded by commenting that "We'd like to encourage a return to thought, experimentation, drawing and testing as a way of discovery and the search for the next great map style. Beck made a cartographic icon for one purpose - to navigate the London Underground; a perfect map made at a perfect place and time. We need new, fresh and challenging maps." This is our attempt to contribute to efforts in this regard.

Our new map undoubtedly shares some characteristics with Beck's original and also with many other versions. This is largely due to the fact that it's the same underlying network. Any solution that seeks to create a diagrammatic version of a transport network will share characteristics and a lineage that extends back to Beck, and others that went before [8]. Yet our map differs from many. Firstly, there's a greater emphasis on the in-between spaces and the above-ground context which we assert is relevant to an individual's wayfinding. Secondly, we're unconstrained from pre-defined design approaches that fit the map to a shape or shapes. Physically sketching (via the peg board) has allowed the map to form organically which we believe overcomes some of the limitations we may have if we over-prescribe graphical demands on structure. Finally, we believe we've made a map that adds a new approach by borrowing from other cartographic work that lends a different aesthetic to the mapping of a transport network. The isometric form of the map portrays the network in a way we've not seen.

Once a final version of the map is complete, our next step is to undergo user testing to explore whether the map has utility beyond an academic and practical exercise. Whether it provides enough utility to offer a genuine alternative is something we are keen to understand, though there have been plenty of very good original designs that have, to our minds, also been far superior to what currently exists $[8,9,10]$. But is the current map so deeply engrained in the psyche of our understanding of transport maps, and in particular, the image of the London Underground map, that this new approach is also doomed to nothing more than an interesting exercise?

What we share is another new design for an old map to contribute to current debates about the enduring legacy of Beck and the need to at least re-think the nature of the map for a modern city and network user $[11,12]$. We should welcome your comments.

[1] K. Field, and W. Cartwright, "Becksploitation: the over-use of a cartographic Icon", The Cartographic Journal, Vol 51, No 4, 343-359, 2014 DOI: 10.1179/0008704114Z.000000000150J

[2] K. Garland, (1994) Mr Beck's underground map, Capital Transport Publishing, Harrow

[3] W. Cartwright, and K. Field, Beck to the future: time to leave it alone. Proceedings of the International Cartographic Conference, Dresden, August 2013

[4] M. Ovenden, Transit maps of the world, Penguin Books, London, 2007

[5] J. Hadlaw, The London Underground Map: imagining modern time and space, Design Issues, Vol 19, No 1, pp 25-35, 2003

[6] M. J. Roberts, Underground maps after Beck, Capital Transport Publishing, Harrow, 2005

[7] K. Field, Cartography., Esri Press, Redlands, 2018

[8] A. Dow, Telling the passenger where to get off: George Dow and the evolution of the railway diagrammatic map, Capital Transport Publishing, Harrow, 2005

[9] M. Noad, London-Tubemap URL: london-tubemap.com, 2012

[10] J. Cerović, Apples to apples: london Underground map update, URL: inat.fr/map/london-underground-map-update, 2018

[11] L. Reynolds, The tube map, redesigned by a Tooting resident, Londonist URL: londonist.com/london/transport/new-alternative-tube-map 2018

[12] M. J. Roberts, Underground maps unravelled, Maxwell Roberts Publishing, Essex, 2012

[13] J. Cerović, One Metro World, J. Cerović, 2016 